\title{
A cross-sectional study for the impact of coping strategies on mental health disorders among psychiatric nurses
}

\author{
Konstantinos Tsaras, ${ }^{1}$ Aristides Daglas, ${ }^{2}$ Dimitroula Mitsi, ${ }^{3}$ Ioanna V. Papathanasiou, ${ }^{1}$ \\ Foteini Tzavella, ${ }^{4}$ Sofia Zyga, ${ }^{4}$ Evangelos C. Fradelos ${ }^{5}$ \\ ${ }^{1}$ Department of Nursing, Technological Educational Institute of Thessaly, Larissa; ${ }^{2}$ Hellenic

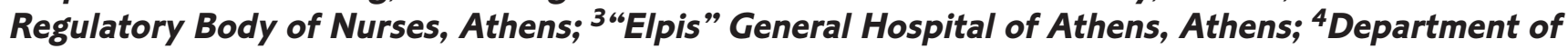 \\ Nursing, University of Peloponnese, Sparta; ${ }^{5}$ Psychiatric Department, "Sotiria" Athens General \\ Hospital for Chest Diseases, Department of Nursing, University of Peloponnese, Sparta, Greece
}

\begin{abstract}
Several studies report that psychiatric nursing is a highly stressful occupation. The ways that nurses use in order to deal with stressful situations have a serious effect on their psychological mood and their health status. The purpose of this study was to investigate the coping strategies in predicting of depression and anxiety among mental health nurses working in public psychiatric hospitals. A descriptive, cross-sectional study was conducted in Athens, Greece from April to May 2017. A questionnaire consisting of the socio-demographic and work-related characteristics, the Patient Health Questionnaire-2, the Generalized Anxiety Disorder-2, and the 38-items Ways of Coping Questionnaire Greek version, was completed by a total of 110 mental health
\end{abstract}

Correspondence: Evangelos C. Fradelos, Psychiatric Department, "Sotiria" Athens General Hospital for Chest Diseases, Department of Nursing, University of Peloponnese, Sparta, Efstathiou \& Stamatikis, Valioti and Plateon, Sparti 23100, Greece.

E-mail: evagelosfradelos@hotmail.com

Key words: Nurses' stress; Depression; Anxiety; Coping strategies; Mental health nurses.

Acknowledgments: the authors would like to thank all staff nurses who participated in the study.

Contributions: KT and ECF jointly initiated the study and planned the research design. KT collected the data and was the primary author of the manuscript, $\mathrm{AD}, \mathrm{DM}, \mathrm{IVP}$ contribute to data collection and writing the draft. KT, ECF FT, and SZ were all engaged in data analysis. All the authors critically read and revised the final version of the manuscript.

Conflict of interest: the authors declare no potential conflict of interest.

Funding: none.

Received for publication: 6 April 2018

Revision received: 5 June 2018.

Accepted for publication: 15 June 2018.

This work is licensed under a Creative Commons AttributionNonCommercial 4.0 International License (CC BY-NC 4.0).

(C) Copyright K. Tsaras et al., 2018

Licensee PAGEPress, Italy

Health Psychology Research 2018; 6:7466

doi:10.4081/hpr.2018.7466 nurses. Univariate and multivariate analyses were performed using the logistic regression model. Coping strategies focused on the problem (positive re-evaluation, positive approach, problem solving, and seeking social support) were the most commonly used by the mental health nurses. Strategies focused on emotion (prayer/daydream, prayer, avoidancelescape, resignation, and denial) were positively associated with depression and anxiety outcomes. Also, the coping skills of problem solving (Adjusted Odds Ratio, AOR=0.402), and seeking social support $(\mathrm{AOR}=3.719)$ were significant predictors only for elevated anxiety symptoms. The results from the study demonstrated the importance of coping behaviours in mental health problems of psychiatric nurses.

\section{Introduction}

Nowadays a growing research interest on mental health and coping in nursing personnel is observed. Nurses is consider to be the most vulnerable working group to experience elevated stress levels due to the fact that are constantly exposed in emotional dementing and challenging situations (Fradelos et al., 2014). These conditions are pouting nurses on an escalating risk to exhibit psychiatric morbidity such anxiety and depression that can affect all aspects of their personal, family and professional life as well (Papathanasiou, 2015).

The are many factors such as individual, demographic, environmental and work related factor that can contribute to the occurrence of mental health issues in nurses (Koinis, Tziaferi \& Saridi, 2014). Important factors for the development and experience of mental disorder among nurses are those related to the very nature of the nursing profession, e.g. daily contact with human pain and end-stage patients, etc., as well as factors related to professional issues e.g. limited means of insufficient nursing potential, lack of training and continuing training, etc. (Antoniou \& Antonodimitrakis, 2001).

Working setting had been proven to be a risk factor for those conditions. Related literature suggesting that nurses working in ICU, Oncologic and psychiatric departments are more likely to experience work related stress levels, anxiety and depression (Fradelos et al., 2014; Papathanasiou et al., 2017; Tsaras et al., 2018). Mental health nursing is an emotional dementing and challenging profession and nurses working in such setting have to deal with many stressors such as the interaction with patients that experiencing mental health issues and their families and the extend social surroundings of them. According to previous researches nurses working in mental health settings are reporting poor mental and physical health as well as poor quality of life. In addition 
those mentioned health parameters can be influenced negatively by the contact with mental illness, workload, high levels of stress, and conflicts with colleagues, supervisors or patient's relatives, the degree of work satisfaction, the balance between work, family and personal development, staff adequacy and salary (Rossi et al., 2012; Hiscott \& Connop, 2009).

All these stressors may lead to losses in job performance, work overload and stress, and interfere with the mental wellbeing of mental health nurses (Ribeiro, Pompeo, Pinto \& Ribeiro, 2015). In addition, by improving working conditions and promoting a less stressing work environment can reduce the incidences of nurse turnover (McGills, 2005; ICN, 2007).

Previous studies are concluding that coping skills are affected by psychological disorders and depressed individuals are using less positive ways of coping. In a recent comparative study among Norwegian and Dutch hospital nurses in which 1814 nurses participated conclude that passive coping style was associated with poor mental health, low job control, low job support and poor physical health (Schreuder et al., 2012). According to the results of a cross sectional study in Hong Kong, positive coping strategies such as positive thinking and help-seeking strategies were associated with less anxiety, depression, and social dysfunction. On the other hand, again passive and negative ways of coping such avoidance are significantly associated with poorer mental health (Wong, Leung \& So, 2001).

Identifying common ways of coping among nurses and training the nurses to use more positive ways to cope with stressors can promote their mental health and wellbeing (Lambert, Lambert \& Ito, 2004; Akbar, Elahi, Mohammadi \& Khoshknab, 2016).

The purpose of this study was to investigate the coping strategies in predicting of depression and anxiety among mental health nurses working in public psychiatric hospitals.

\section{Materials and Methods}

\section{Study design and sample}

This study utilized a descriptive, cross-sectional research design. The source population was all mental health nurses who were working in public psychiatric hospitals of Greece. The sample consisted of 110 mental health nurses who were randomly selected from two public psychiatric hospitals in Athens of Greece. Stratified random sampling procedure per hospital was used in recruiting samples. The sample size is approximately $10 \%$ of the source population's nurses. Nurses were chosen using the following criteria: i) being a nurse or nurse assistant with immediate association with patients, ii) having working experience in nursing at least 1 year, iii) having adequate knowledge of the Greek language and satisfactory level of communication, and iv) being consented to participate in the study. Out of 150 nurses, 110 returned the study questionnaire (response rate $=73.3 \%$ ).

\section{Research instruments}

Data were collected using a self-reporting and structured questionnaire, during the period of April 2017 and May 2017. The questionnaire consisted of three parts:

i) Individual characteristics. The basics socio-demographic and professional characteristics (gender, age, marital status, educational level, working experience in nursing, working position, and work shift) of the nurses were recorded.

ii) Patient Health Questionnaire-2 (PHQ-2) and Generalized
Anxiety Disorder-2 (GAD-2) scales. The PHQ-2 and the GAD-2 questionnaire were produced as ultra-brief screening instruments for depression and anxiety, suitable for use in epidemiological studies. The PHQ-2 includes two questions and it was found to have good sensitivity and specificity for detecting depressive disorders (Kroenke et al., 2003). Likewise, the GAD-2 questionnaire which comprises two questions appears to have acceptable accuracy for detecting generalized anxiety, panic, social anxiety and post-traumatic stress disorder (Kroenke et al., 2007). In both questionnaires, each question requires respondents to rate on a four-point scale ranging from " $0=$ not at all" to " 3 = nearly every day". PHQ-2 and GAD-2 total scores are calculated by adding the two questions score, resulting in a range from 0 to 6 for each questionnaire, with higher score indicative of higher mental health disorder. According to receiver-operating characteristic curve analysis, the optimal cutpoint is $\geq 3$ on both the PHQ-2 and GAD-2 scales (Löwe et al., 2010; Kroenke et al., 2010).

iii) Ways of Coping Questionnaire (WCQ). The Greek version of the WCQ (revised) was used to explore the coping strategies (Karadimas, 1998). The WCQ was developed by Folkman et al. and encompasses thoughts and actions that people use to deal with internal or external demands of a specific stressful event (Folkman et al., 1986). The instrument after translation and validation in Greek language consists of 38 items which are responded to on a four-point Likert scale ranging from " 0 $=$ never" to " 3 = often". The items are divided into five factors: i) Positive approach (11 items), including positive re-evaluation and problem solving, ii) Seeking social support (6 items), iii) Prayer/Daydream (8 items), including prayer and searching for divine intervention, iv) Avoidance/Escape (9 items), including resignation and denial, and v) Assertive problem solving (4 items). The scale does not show the total score as sum for evaluation, since the items should be assessed by the mean scores of each factor. Higher score indicates that the coping strategy is regularly used. In the original study and according to psychometric analysis of the WCQ - Greek version, the internal consistency reliability of eleven coping strategy factors, measured by Cronbach's alpha coefficient ranged from 0.60 to 0.79 .

\section{Ethical considerations}

The study protocol was approved by the Ethics Committee and the Scientific Council of all participating hospitals. The research was executed following the principles of confidentiality, anonymity and informed consent, as outlined by the Declaration of Helsinki and its subsequent revisions.

\section{Statistical analysis}

Means and standard deviations were calculated to describe continuous variables. The categorical variables were presented as absolute (n) and relative (\%) frequencies. The occurrences of depression and anxiety were used as the outcomes (dependent variables) of the under-research correlations. The association between ways of coping strategies and mental health disorders was examined using univariate logistic regression analyses. In order to investigate the effect of coping strategies on depression and anxiety multiple logistic regression analysis was conducted after adjusting for gender, age, marital status, educational level, working experience, working position, and work shift. Odds ratio with $95 \%$ confidence interval was used as measure of association. All reported p-values were two-tailed, and a p-value under 0.05 was consid- 
ered statistically significant in all analyses. Statistics of the research's empirical data were processed with IBM SPSS for Windows (version 21.0, SPSS Inc., Chicago, IL, USA).

\section{Results}

\section{Socio-demographic and professional characteristics}

A total of 110 psychiatric nurses were included in this study. Most participants were women, married and had secondary education. The majority were nursing assistants and worked on rotating shifts. The socio-demographic and professional characteristics of the nurses participated in the study are shown in Table 1.

\section{Depression and anxiety}

The mean total score of PHQ-2 and GAD-2 questionnaire was $2.57(\mathrm{SD}=1.82)$ and $2.66(\mathrm{SD}=1.90)$, respectively. According to the cut off points of depression (PHQ-2 score $\geq 3$ ) and anxiety (GAD-2 score $\geq 3$ ), a high percentage of the mental health nurses were at risk for psychiatric disorder. Specifically, the screening method showed prevalence 52.7\% (58/110) for depression and $48.2 \%(53 / 110)$ for anxiety.

\section{Ways of coping strategies}

Reliability and descriptive statistical measures of the WCQ Greek version are presented in Table 2 . The reliability was determined by assessing the Cronbach's alpha (internal consistency evaluation of data). The WCQ - Greek version showed a moderate to high internal consistency reliability. Based upon the total score and the number of questions, the mean score that was given by each nurse respondent was computed. The mean scores of WCQ factors indicated that strategies of positive re-evaluation, positive approach, problem solving, and seeking social support (so called positive or problem-focused forms of coping) were the most commonly used by the mental health nurses, while avoidance/escape, resignation, prayer/daydream, and searching for divine intervention (so called negative or emotional-focused types of coping) were the lowest ranked coping skills.

\section{Association between coping strategies and depression}

A logistic regression analysis was performed to explore the relationship between depression and WCQ factors. Table 3 presents both the crude and the adjusted odds ratios for depressive symptoms in ways of coping strategies after controlling for sociodemographic and work-related characteristics. Specifically, age and years of working experience in nursing were significantly positively associated with the risk of depression. In addition, the risk of depression development was higher in single and divorced/widowed nurses than married nurses, tertiary education nurses than secondary education nurses, and nurses than nursing assistants.

The results showed that all coping strategies focused on emotion, except searching of divine intervention, were significant predictors of increased depression risk. In particularly, were found positive associations between prayer/daydream, prayer, avoidancelescape, resignation, denial and elevated depression symptoms among mental health nurses. Also, problem-focused forms of

Table 1. Mental health nurses' characteristics ( $n=110)$.

\begin{tabular}{lrr} 
Characteristics & n & \\
Gender & & \\
Male & 39 & $(35.5 \%)$ \\
Female & 71 & $(64.5 \%)$ \\
Age (years), mean \pm sd & $42.64 \pm 5.87$ \\
Marital status & & \\
$\quad$ Married & 65 & $(59.1 \%)$ \\
Single & 30 & $(27.3 \%)$ \\
Divorced/Widowed & 15 & $(13.6 \%)$ \\
\hline Educational level & & \\
High school & 57 & $(51.8 \%)$ \\
University/Technical university & 35 & $(31.8 \%)$ \\
Postgraduate degree & 18 & $(16.4 \%)$ \\
Working experience in nursing (years), mean \pm sd & $15.73 \pm 5.64$ \\
Working position & & \\
$\quad$ Nurse assistant & 59 & $(53.6 \%)$ \\
Nurse & 41 & $(37.3 \%)$ \\
Head of department & 10 & $(9.1 \%)$ \\
Work shift & & \\
Rotated & 95 & $(86.4 \%)$ \\
Morning & 15 & $(13.6 \%)$ \\
\hline
\end{tabular}

Table 2. Ways of coping questionnaire (38-items Greek version).

\begin{tabular}{lccccc}
\hline Factors of WCQ & Item amount & Factor ranking & Mean \pm SD & Median & Cronbach's alpha \\
1. Positive approach & 11 & 2 & $1.925 \pm 0.600$ & 1.909 & 0.909 \\
1.a Positive re-evaluation & 7 & 1 & $1.957 \pm 0.625$ & 1.857 & 0.865 \\
\hline 1.b Problem solving & 4 & 3 & $1.868 \pm 0.663$ & 1.875 & 0.840 \\
2. Seeking social support & 6 & 4 & $1.847 \pm 0.648$ & 1.833 & 0.838 \\
\hline 3. Prayer/Daydream & 8 & 10 & $1.589 \pm 0.637$ & 1.625 & 0.850 \\
3.a Prayer & 5 & 5 & $1.738 \pm 0.693$ & 1.800 & 0.829 \\
\hline 3.b Searching of divine intervention & 3 & 11 & $1.339 \pm 0.772$ & 1.333 & 0.767 \\
4. Avoidance/ Escape & 9 & 8 & $1.670 \pm 0.608$ & 1.722 & 0.853 \\
\hline 4.a Resignation & 5 & 9 & $1.649 \pm 0.659$ & 1.600 & 0.786 \\
4.b Denial & 4 & 6 & $1.696 \pm 0.646$ & 1.750 & 0.691 \\
\hline 5. Assertive problem solving & 4 & 7 & $1.675 \pm 0.645$ & 1.750 & 0.647 \\
\hline 4-pont Likert scale was used $(0=$ never to $3=$ often) & & & &
\end{tabular}


coping were not associated with significantly risk for depressive disorder in psychiatric nurses.

\section{Association between coping strategies and anxiety}

Table 4 presents simple and multiple logistic regressions of the WCQ factors against the risk of anxiety symptoms. The odds ratios were adjusted for socio-demographic and work-related characteristics. Particularly, age of nurses and working experience in nursing were positive predictors of anxiety disorder. Also, anxiety risk was more frequent in single nurses than married nurses, tertiary education nurses and nurses with a postgraduate degree than secondary education nurses, nurses and heads of departments than nursing assistants.

According to the statistical results, the relations that were found between anxiety and coping strategies focused on emotion were similar with the relations that were found between depression and coping strategies focused on emotion. Specifically, prayer/daydream, prayer, avoidance/escape, resignation, and denial predicted increased possibility for anxiety disorder. In addition, an interesting finding about problem-focused forms of coping is that problem solving had significant negative association with anxiety, and seeking social support had significant positive association with anxiety risk among psychiatric nurses.

\section{Discussion}

The purpose of this study was to investigate the coping strategies in predicting of depression and anxiety among mental health nurses working in public psychiatric hospitals. According to our results a high percentage of mental health nurses are in high risk for developing anxiety or depression. Specifically, we found that $52.7 \%$ of the nurses were suffering from depression and $48.2 \%$ from anxiety. Our results are in accordance with previous studies, which showed higher rates of depressive symptoms (Kourakos, 2017; Letvak, Ruhm \& McCoy, 2012; Shahri et al., 2017) and anxiety (De Souza et al., 2017; Kourakos, 2017) among nursing professionals. Jordan et al. (2016) who examined the impact of perceived stress and coping adequacy on the health of nurses, also found that nurses were not healthy, as $92 \%$ had moderate-to-very

Table 3. Simple and multiple logistic regression results with depression as dependent variable and WCQ factors as independent, adjusted for socio-demographic and work-related characteristics.

\begin{tabular}{lccccc} 
Factors of WCQ & \multicolumn{2}{c}{ Depression (PHQ-2 $\geq 3)$} & COR $(95 \%$ CI $)$ & AOR (95\% CI) \\
Positive approach & $1.936 \pm 0.394$ & No $(\mathrm{n}=52)$ & $1.913 \pm 0.772$ & $1.067(0.570,1.998)$ & $1.194(0.531,2.685)$ \\
Positive re-evaluation & $1.985 \pm 0.423$ & $1.926 \pm 0.796$ & $1.166(0.638,2.131)$ & $1.542(0.701,3.396)$ \\
\hline Problem solving & $1.849 \pm 0.475$ & $1.889 \pm 0.829$ & $0.912(0.517,1.609)$ & $0.749(0.353,1.589)$ \\
Seeking social support & $1.828 \pm 0.510$ & $1.869 \pm 0.778$ & $0.906(0.506,1.621)$ & $2.269(0.964,5.341)$ \\
\hline Prayer/Daydream & $1.726 \pm 0.582$ & $1.435 \pm 0.666$ & $2.135(1.134,4.021)^{*}$ & $2.548(1.144,5.676)^{*}$ \\
Prayer & $1.928 \pm 0.570$ & $1.527 \pm 0.759$ & $2.480(1.349,4.557)^{* *}$ & $2.558(1.216,5.383)^{*}$ \\
\hline Searching of divine intervention & $1.391 \pm 0.847$ & $1.282 \pm 0.681$ & $1.204(0.737,1.966)$ & $1.605(0.820,3.142)$ \\
Avoidance / Escape & $1.891 \pm 0.442$ & $1.423 \pm 0.675$ & $4.557(2.049,10.13)^{* *}$ & $3.667(1.429,9.409)^{* *}$ \\
\hline Resignation & $1.879 \pm 0.498$ & $1.392 \pm 0.723$ & $3.684(1.819,7.462)^{* *}$ & $3.252(1.379,7.669)^{* *}$ \\
Denial & $1.905 \pm 0.504$ & $1.462 \pm 0.709$ & $3.308(1.665,6.571)^{* *}$ & $2.719(1.189,6.218)^{*}$ \\
\hline Assertive problem solving & $1.716 \pm 0.539$ & $1.630 \pm 0.748$ & $1.232(0.685,2.216)$ & $1.596(0.753,3.384)$ \\
\hline
\end{tabular}

Data shown as mean \pm sd. $W C Q=$ Ways of Coping Questionnaire, $\mathrm{PHQ}=$ Patient Health Questionnaire, $\mathrm{COR}=$ Crude Odds Ratio, AOR $=$ Adjusted Odds Ratio. ${ }^{*} \mathrm{P}<0.05{ }^{*} * \mathrm{P}<0.01$.

Table 4. Simple and multiple logistic regression results with anxiety as dependent variable and WCQ factors as independent, adjusted for socio-demographic and work-related characteristics.

\begin{tabular}{|c|c|c|c|c|}
\hline Factors of WCQ & $\begin{array}{r}\text { An } \\
\text { Yes }(n=53)\end{array}$ & No $(n=52)$ & COR $(95 \% \mathrm{CI})$ & AOR (95\% CI) \\
\hline Positive approach & $1.877 \pm 0.387$ & $1.970 \pm 0.746$ & $0.769(0.409,1.447)$ & $0.668(0.312,1.431)$ \\
\hline Positive re-evaluation & $1.943 \pm 0.419$ & $1.970 \pm 0.773$ & $0.934(0.512,1.704)$ & $0.970(0.477,1.974)$ \\
\hline Problem solving & $1.759 \pm 0.444$ & $1.969 \pm 0.807$ & $0.611(0.339,1.100)$ & $0.402(0.192,0.839)^{*}$ \\
\hline Seeking social support & $1.934 \pm 0.497$ & $1.766 \pm 0.757$ & $1.511(0.831,2.748)$ & $3.719(1.504,9.192)^{* *}$ \\
\hline Prayer/Daydream & $1.726 \pm 0.563$ & $1.461 \pm 0.679$ & $1.991(1.064,3.724)^{*}$ & $2.120(1.028,4.373)^{*}$ \\
\hline Prayer & $1.928 \pm 0.544$ & $1.561 \pm 0.770$ & $2.290(1.255,4.179)^{* *}$ & $2.253(1.125,4.514)^{*}$ \\
\hline Searching of divine intervention & $1.390 \pm 0.816$ & $1.292 \pm 0.732$ & $1.180(0.724,1.925)$ & $1.377(0.772,2.457)$ \\
\hline Avoidance/Escape & $1.891 \pm 0.434$ & $1.464 \pm 0.675$ & $3.892(1.800,8.414)^{* *}$ & $2.671(1.171,6.095)^{*}$ \\
\hline Resignation & $1.876 \pm 0.458$ & $1.439 \pm 0.746$ & $3.152(1.594,6.233)^{* *}$ & $2.447(1.154,5.191)^{*}$ \\
\hline Denial & $1.910 \pm 0.519$ & $1.496 \pm 0.692$ & $3.045(1.546,5.995)^{* *}$ & $2.127(1.015,4.454)^{*}$ \\
\hline Assertive problem solving & $1.750 \pm 0.580$ & $1.605 \pm 0.697$ & $1.428(0.788,2.586)$ & $1.595(0.795,3.200)$ \\
\hline
\end{tabular}

Data shown as mean \pm Sd. $W C Q=$ Ways of Coping Questionnaire, $\mathrm{GAD}=$ Generalized Anxiety Disorder, $\mathrm{COR}=$ Crude Odds Ratio, $\mathrm{AOR}=$ Adjusted $0 \mathrm{dds}$ Ratio. ${ }^{*} \mathrm{P}<0.05{ }^{*} * \mathrm{P}<0.01$ 
high stress levels, $78 \%$ slept less than the recommended eight hours a night and $22 \%$ were classified as binge drinkers (Jordan et $a l, 2016)$. Nurses have one of the most important jobs within hospitals and have some of the highest levels of direct patient contact than do any other health care professions. Mental health disorders are life-threatening situations that have profound effects on an individual's social well-being, which render nursing in inpatient psychiatric settings complex and challenging (Hasan, 2017). Also, mental health professions involve peculiarities that expose them to health problems, psychological imbalances and a low degree of quality of life (Depastas \& Pierrakos, 2015). The physical and mental health of psychiatric nurses is significantly affected by factors such as contact with the mentally ill, heavy workload, continuous stress, organizational structure, home/work and inter professional conflicts, levels of degrees of job satisfaction, work-life balance, staffing adequacy and low salaries which lead to feelings of depression, helplessness and hopelessness (Depastas \& Pierrakos, 2015; Abdalrahim, 2013). The increasing demands faced by nurses impact all areas of their personal and professional lives and increase their risks of chronic stress and unhealthy behaviours. Psychiatric morbidity is gradually phased out, resulting in chronic mental health disorders among healthcare professionals, such as burnout and depression that can impact all areas of life (Koinis et al., 2014). All those mentioned above can be possible explanations of our results and explain the high rates of depression and anxiety among our participants.

Present study found high mean score for positive or problemfocused forms of coping, with the strategies of positive re-evaluation, positive approach, problem solving, and seeking social support being the most commonly used by the mental health nurses. Our findings agree with those of a similar research study conducted by Jose \& Bhat (2013) where nurses used positive reappraisal and social support to cope with job stress (Jose \& Bhat, 2013) and with the one conducted by Ribeiro et al. (2015) who found that the strategies of confrontation and distancing were less commonly used by the nursing professionals in the studied care units (Ribeiro et al., 2015). On the other hand our findings regarding coping strategies are opposite to that of McTiernan \& McDonald (2015) who found that avoidance coping and more specifically the strategy of Diverting one's attention was the most frequently used by psychiatric nurses in their study (McTiernan \& McDonald, 2015).

Mental health nurses need to deal with their stress in order to ensure every patient receives the best care available. It is consequently, important for them to cultivate good health, stimulating the use of several strategies that strengthen their ability to cope effectively. This, in turn, will reduce the levels of stress and burnout they experience. Understanding the way mental health nurses cope with work-related stress is an important workplace strategy, for the nurses, for their organizations and the recipients of their care (AL-Sagarat, 2017). The combination of individual coping skills, effective organizational planning and social support are the best and most effective ways to manage and/or cope with stressful situations in nurses (Iyi, 2015). Coping strategies used by nurses at work may include seeking support from colleagues or formal support, planful problem solving and avoidance of painrelated stimuli (Akuroma et al., 2016). Especially, the most widely used strategies, by nurses, include the positive strategic approach and positive reassessment, problem based solution, planning work and priority setting in the work while less frequently used ways are those focused on emotion such as avoidance, humor, refusal, selfblame and acceptance of responsibilities. Self-control has also been a stress coping strategy according to bibliography, as well as passive strategies such as invoking to God which have been report- ed less frequently (Zyga et al., 2016).

The present study found that emotion-focused coping, except searching of divine intervention, was associated with an increased risk for depression and anxiety disorders among mental health nurses. These results are in agreement with other research which suggest that coping strategies that are more problem-focused, rather than emotion-focused, tend to be associated with better mental health when dealing with workplace stress (Laranjeira, 2012, Healy \& McKay 2000).

In our study, less anxiety symptoms were also associated with problem-focused forms of coping, as problem solving (Adjusted Odds Ratio, AOR $=0.402,95 \%$ CI: $0.192-0.839$ ) was found to have significant negative association with anxiety, while seeking social support $(\mathrm{AOR}=3.719,95 \% \mathrm{CI}$ : 1.504 - 9.192) were significantly associated with increased risk of anxiety among psychiatric nurses. Similar to our results, Boumans (1992), also found that use of problem $\square$ focused coping was positively associated with job satisfaction, and negatively related to health complaints (Boumans \& Landeweerd, 1992). Finally, Mark \& Smith (2012) discovered robust associations between coping strategies, job demands, levels of control, social support, anxiety and depression in nurses, with social support, rewards, and skill discretion being negatively associated with mental health problems (Mark \& Smith, 2012).

This study was an attempt to assess for first time depression and anxiety using subjective ultra-brief measures and their association with coping strategies among Greek mental health nurses. Moreover, to our best of knowledge there is a lack of research on assessing the relation of mental health and coping strategies among mental health nurses. Regarding the limitations of the study it is noted that that interference of external variables (interruption by others, noise level and fatigue of the participants cause most of respondents completed the questionaries' during their shift), probably influenced the answers of respondents. In addition, the crosssectional design of our study, that does not allow conclusions about causality of depression and anxiety among mental health nurses. Results cannot be generalized for all Greek mental health nurses as the study was conducted in Athens region even if this area is a major geographic regions of Greece. Therefore, more studies assessing the relation between mental health and coping strategies among mental health nurses are needed.

\section{Conclusions}

Stress and other mental health issues are in high prevalence in caring profession and especially in mental health nursing. The extend workload, the constant interaction with people that are suffering and the exposure to aggressiveness are some of the factors contributing to this and making it a global phenomenon. Coping strategies can effect mental health of psychiatric nurses and the identification of strategies that are used and the promotion of positive strategies are two issues of high importance in mental health nursing. There is an urgent need to promote research in this area for recognizing effective strategies to prevent stress and cope with it. Establishing and implementing effective policies in work places, well-organized and democratic administration, may contribute to addressing the existing difficulties more effectively. Moreover, implementing intervention for promoting mental and physical health, psychological support groups and training in problem solving technics may be proven effective in coping among mental health nurses. 


\section{References}

Abdalrahim, A. A. (2013). Stress and coping among psychiatric nurses. Middle East Journal of Nursing, 7(4), 30-37.

Akuroma, R., Eye, A., \& Curran, T. (2016). Coping strategies used by nurses in dealing with patient death and dying. Bachelor's thesis. Degree Programme in Nursing. Social services, Health \& Sport. JAMK University of Applied Sciences, Finland.

AL-Sagarat, A. Y., Barmawi, M., Al Hadid, L. A., Qaddumi, J. A., \& Moxham, L. (2017). Validating the psychiatric nurses methods of coping questionnaire: Arabic version. BMC Psychiatry, 17(1), 410.

Antoniou, A. S., \& Antonodimitrakis, P. (2001). Levels of job satisfaction and coping strategies of occupational stress used by Greek hospital doctors. In: Proceedings of the 8th Greek Conference of Psychological Research, Alexandroupoli (Vol. 72).

Boumans, N. P. G., \& Landeweerd, J. A. (1992). The role of social support and coping behaviour in nursing work: main or buffering effect? Work \& Stress, 6(2), 191-202.

Depastas, C., \& Pierrakos, G. (2015). The mental health of mental health professionals of non-governmental organizations in the national program "Psychargos": The effects of the economic crisis. Archives of Hellenic Medicine, 32(6), 758-765.

Akbar, R. E., Elahi, N., Mohammadi, E., \& Khoshknab, M. F. (2016). What strategies do the nurses apply to cope with job stress?: a qualitative study. Global Journal of Health Science, $8(6), 55$.

Folkman, S., Lazarus, R. S., Dunkel-Schetter, C., DeLongis, A., \& Gruen, R. J. (1986). Dynamics of a stressful encounter: cognitive appraisal, coping, and encounter outcomes. Journal of Personality and Social Psychology, 50(5), 992.

Fradelos, E., Tzitzikos, G., Giannouli, V., Argyrou, P., Vassilopoulou, C., \& Theofilou, P. (2014). Assessment of burnout and quality of life in nursing professionals: the contribution of perceived social support. Health Psychology Research, 2(1).

Hasan, A. A. (2017). Work stress, coping strategies and levels of depression among nurses working in mental health hospital in port-said city. International Archives of Nursing and Health Care, 3(2), 1-10.

Healy, C. M., \& McKay, M. F. (2000). Nursing stress: the effects of coping strategies and job satisfaction in a sample of Australian nurses. Journal of Advanced Nursing, 31(3), 681688.

Hiscott, R. D., \& Connop, P. J. (1996). Why hospital mental health staff terminate employment. Perspectives in Psychiatric Care, 32(1), 7-11.

International Council of Nurses (2007). Positive Practice Environments: Quality Workplace-Quality Patient Care. Geneva: ICN.

Iyi, O. (2015). Stress Management and Coping Strategies among Nurses: A Literature Review. Degree Thesis. Degree Programme in Nursing. Lovisa City, Finland.

Jordan, T. R., Khubchandani, J., \& Wiblishauser, M. (2016). The impact of perceived stress and coping adequacy on the health of nurses: a pilot investigation. Nursing Research and Practice, 1-11.

Jose, T. T., \& Bhat, S. M. (2013). A descriptive study on stress and coping of nurses working in selected hospitals of Udupi and Mangalore districts Karnataka, India. IOSR Journal of Nursing and Health Science, 3(1), 10-18.
Karadimas, E. C. (1998). The adaptation of the ways of coping questionnaire in the Greek language. Psychology, 5, 260-273.

Koinis, Ar., Tziaferi, St., \& Saridi, M. (2014). Mental health problems in health professionals. Interscientific Health Care, 6(1), 8-17.

Kourakos, M., (2017). Mental health and coping strategies among nursing staff in public health services. Progress in Health Sciences, 7, 67-73.

Kroenke, K., Spitzer, R. L., \& Williams, J. B. (2003). The Patient Health Questionnaire-2: validity of a two-item depression screener. Medical Care, 41(11), 1284-1292.

Kroenke, K., Spitzer, R. L., Williams, J. B., \& Löwe, B. (2010). The patient health questionnaire somatic, anxiety, and depressive symptom scales: a systematic review. General Hospital Psychiatry, 32(4), 345-359.

Kroenke, K., Spitzer, R. L., Williams, J. B., Monahan, P. O., \& Löwe, B. (2007). Anxiety disorders in primary care: prevalence, impairment, comorbidity, and detection. Annals of Internal Medicine, 146(5), 317-325.

Lambert, V. A., Lambert, C. E., \& Ito, M. (2004). Workplace stressors, ways of coping and demographic characteristics as predictors of physical and mental health of Japanese hospital nurses. International Journal of Nursing Studies, 41(1), 85-97.

Laranjeira, C. A. (2012). The effects of perceived stress and ways of coping in a sample of Portuguese health workers. Journal of Clinical Nursing, 21(11 $\square 12)$, 1755-1762.

Letvak, S., Ruhm, C. J., \& McCoy, T. (2012). Depression in hospital-employed nurses. Clinical Nurse Specialist, 26(3), 177182.

Löwe, B., Wahl, I., Rose, M., Spitzer, C., Glaesmer, H., Wingenfeld, K., ... \& Brähler, E. (2010). A 4-item measure of depression and anxiety: validation and standardization of the Patient Health Questionnaire-4 (PHQ-4) in the general population. Journal of Affective Disorders, 122(1), 86-95.

Mark, G., \& Smith, A. P. (2012). Occupational stress, job characteristics, coping, and the mental health of nurses. British Journal of Health Psychology, 17(3), 505-521.

McGills Hall, L. M. (2005). Quality work environments for nurse and patient safety. Sudbury, MS: Jones \& Bartlett Learning.

McTiernan, K., \& McDonald, N. (2015). Occupational stressors, burnout and coping strategies between hospital and community psychiatric nurses in a Dublin region. Journal of Psychiatric and Mental Health Nursing, 22(3), 208-218.

Papathanasiou, I. V. (2015). Work-related mental consequences: Implications of burnout on mental health status among health care providers. Acta Informatica Medica, 23(1), 22-28.

Papathanasiou, I. V., Tsaras, K., Kleisiaris, C. F., Fradelos, E. C., Tsaloglidou, A., \& Damigos, D. (2017). Anxiety and depression in staff of mental units: The role of burnout. In GeNeDis 2016 (pp 185-197). Springer; Cham.

Ribeiro, R. M., Pompeo, D. A., Pinto, M. H., \& Ribeiro, R. D. C. H. M. (2015). Estratégias de enfrentamento dos enfermeiros em serviço hospitalar de emergência. Acta Paulista de Enfermagem, 28(3), 216-223.

Rossi, A., Cetrano, G., Pertile, R., Rabbi, L., Donisi, V., Grigoletti, L., ... \& Amaddeo, F. (2012). Burnout, compassion fatigue, and compassion satisfaction among staff in community-based mental health services. Psychiatry Research, 200(2), 933-938.

Schreuder, J. A., Roelen, C. A., Groothoff, J. W., van der Klink, J. J., Magerøy, N., Pallesen, S., ... \& Moen, B. E. (2012). Coping styles relate to health and work environment of Norwegian and Dutch hospital nurses: A comparative study. Nursing Outlook, 60(1), 37-43. 
Shahri, S., Sadat, S., Ghashghaee, A., Behzadifar, M., Luigi Bragazzi, N., Behzadifar, M., ... Seyedin, H. (2017). Depression among Iranian nurses: A systematic review and meta-analysis. Medical Journal of The Islamic Republic of Iran (MJIRI), 31(1), 860-868.

Tsaras, K., Papathanasiou, I.V., Vus, V., Panagiotopoulou, A., Katsou, M.A, Kelesi, M., Fradelos, E.C. (2018). Predicting Factors of Depression and Anxiety in Mental Health Nurses: A Quantitative Cross-Sectional Study. Medical Archives, 72, 62-
67.

Wong, D., Leung, S., So, C. (2001). Differential impacts of coping strategies on the mental health of Chinese nurses in hospitals in Hong Kong. International Journal of Nursing Practice, 7, 188-198.

Zyga, S., Mitrousi, S., Alikari, V., Sachlas, A., Stathoulis, J., Fradelos, E., Panoutsopoulos, G., Maria, L. (2016). Assessing factors that affect coping strategies among nursing personnel. Materia Sociomedica, 28, 146-150. 